\title{
BMJ Open Evaluating phone camera and cloud service-based 3D imaging and printing of human bones for anatomical education
}

\author{
Qing-Yun Li, ${ }^{1,2}$ Qi Zhang (D , ${ }^{1}$ Chun Yan, ${ }^{1}$ Ye He, ${ }^{3}$ Mukuze Phillip, ${ }^{2}$ Fang Li, ${ }^{1}$ \\ Ai-Hua Pan ${ }^{1}$
}

To cite: Li Q-Y, Zhang Q, Yan C, et al. Evaluating phone camera and cloud service-based $3 \mathrm{D}$ imaging and printing of human bones for anatomical education. BMJ Open

2020;10:e034900. doi:10.1136/ bmjopen-2019-034900

- Prepublication history and additional material for this paper are available online. To view these files, please visit the journal online (http://dx.doi. org/10.1136/bmjopen-2019034900).

$Q-Y L$ and $Q Z$ contributed equally.

Received 11 October 2019 Revised 15 January 2020 Accepted 15 January 2020

Check for updates

(C) Author(s) (or their employer(s)) 2020. Re-use permitted under CC BY-NC. No commercial re-use. See rights and permissions. Published by BMJ.

${ }^{1}$ Department of Human Anatomy and Neurobiology, and Morphologic Science Experimental Center, School of Basic Medical Science, Central South University, Changsha,

China

${ }^{2}$ Class of 2020, Xiangya School of Medicine, Central South University, Changsha, Hunan, China

${ }^{3}$ Aier School of Ophthalmology, Central South University, Changsha, China

Correspondence to

Dr Ai-Hua Pan;

panaihua@csu.edu.cn

\section{ABSTRACT}

Objective To evaluate the feasibility of a phone camera and cloud service-based workflow to image bone specimens and print their three-dimensional (3D) models for anatomical education.

Design The images of four typical human bone specimens, photographed by a phone camera, were aligned and converted into digital images for incorporation into a digital model through the Get3D website and submitted to an online 3D printing platform to obtain the 3D printed models. The fidelity of the 3D digital, printed models relative to the original specimens, was evaluated through anatomical annotations and 3D scanning. Setting The Morphologic Science Experimental Center, Central South University, China.

Participants Specimens of four typical bones-the femur, rib, cervical vertebra and skull-were used to evaluate the feasibility of the workflow.

Outcome measures The gross fidelity of anatomical features within the digital models and 3D printed models was evaluated first using anatomical annotations in reference to Netter's Atlas of Human Anatomy. The measurements of the deviation were quantised and visualised for analysis in Geomagic Control 2015.

Results All the specimens were reconstructed in $3 \mathrm{D}$ and printed using this workflow. The overall morphology of the digital and 3D printed models displayed a large extent of similarity to the corresponding specimens from a gross anatomical perspective. A high degree of similarity was also noticed in the quantitative analysis, with distance deviations $\leq 2 \mathrm{~mm}$ present among $99 \%$ of the random sampling points that were tested.

Conclusion The photogrammetric digitisation workflow adapted in the present study demonstrates fairly high precision with relatively low cost and fewer equipment requirements. This workflow is expected to be used in morphological/anatomical science education, particularly in institutions and schools with limited funds or in certain field research projects involving the fast acquisition of $3 \mathrm{D}$ digital data on human/animal bone specimens or on other remains.

\section{INTRODUCTION}

Full-body dissection has served as a 'gold standard' in anatomic science education
Strengths and limitations of this study

- This study provides a possible means for medical students and teachers to build three-dimensional (3D) digital or printing models for use in anatomical education.

- By dividing the femur model into cross sections, the 3D model can maintain a certain size of corresponding specimens and the problem of printer adaption could possibly be addressed.

- Using phone camera-based photogrammetry, cloud processing services for 3D modelling, and the hollowing method to control the use of resins, this study has tried to make the $3 \mathrm{D}$ printing of bone specimens more cost-effective.

- The photogrammetry-based method does not capture the internal structure of the bone specimens.

- This workflow is not applicable to medical schools in the countries that have no internet connection, although the dataset needed to upload to cloud services is not large.

for centuries, ${ }^{12}$ as direct cadaver dissection facilitates the observation and exploration of anatomical details and provides an experience of tactile sensation comparable to the physical examination of a living body. However, owing to the insufficiency of donated bodies and the high cost of maintaining cadavers, the availability of real human cadavers for medical students worldwide is low. ${ }^{3-6}$ Thanks to progress achieved in multimedia technology, new teaching/learning methods have emerged and have been evaluated as substitutes for the traditional cadaver-based medical training in the recent years. ${ }^{7}$ The three-dimensional (3D) printing represents a major trend in this direction. ${ }^{8-10}$

3D printing, also known as rapid prototyping or additive manufacturing, is a computer-mediated technology that creates 3D products directly from 3D modelling data by sequential layer-by-layer deposition of 
material. ${ }^{11}$ The primary advantage of $3 \mathrm{D}$ printing lies in its ability to create graspable shapes or geometric features of high complexity, overcoming the limitations brought about by the use of flat screens for the visualisation of 3D imaging data. Moreover, compared with embalmed cadaveric specimens, 3D printed models are more wearresistant, easier to clean and store, ${ }^{12}$ and, essentially, environmentally green. ${ }^{5} 1012$

The applications of 3D printing are expanding rapidly. ${ }^{13}$ In clinical practice, 3D printing is reported to have been used in a variety of ways, including, but not limited to, planning surgeries, simulating operations, preoperative conversation between doctors and patients, and fabricating customised and personalised equipment during the recovery stage. ${ }^{514-17}$ As far as anatomical education is concerned, 3D printing is also playing an increasingly significant role in some developing countries such as India $^{18}$ and Brazil. ${ }^{19}$ 3D printing can be used to make replicas of separate anatomical structures ${ }^{20}$ or multiple structures combined to display the spatial relationship between them. ${ }^{21} 3 \mathrm{D}$ replicas are also well qualified to illustrate clinicoanatomical structures. ${ }^{22}$ In effect, reports indicate that 3D printing helps to improve the effectiveness of teaching ${ }^{23}$ and that students learning with 3D printed models performed even better in tests than those learning with real specimens. ${ }^{20}$

At present, there are a large number of 3D printers based on various printing technologies. From these available printers, ceramic, metal, wax and resin materials can be used to print digital documents using highly unique processes, depending on the techniques used. ${ }^{24}$ Generally, 3D printing techniques include fused deposition modelling (FDM), selective laser melting/selective laser sintering (SLS), multijet modelling, laminated object manufacturing (LOM) and stereolithography apparatus (SLA), which is a new technique that uses an ultraviolet laser to convert a liquid polymer into a solid resin. ${ }^{25} 26$

Despite the potential and merits, 3D printing can also be a challenge for non-specific practitioners. Since all 3D printed models are fabricated based on the 3D digital model, which is created by $3 \mathrm{D}$ digitisation and serves as a blueprint, the methods of 3D digitisation significantly impact the whole 3D printing workflow as well as the quality of 3D printed models.

3D digitisation is a process of transforming physical objects into digital models. Several methods have been used to fabricate medical digital models. Depending on how the data are acquired, they can be divided into several main types: (1) patient-specific digital imaging and communication in medicine (DICOM) images based on CT/MRI/ultrasound dataset, (2) specimen data derived from scanning with specific 3D scanner ${ }^{27} 28$ and (3) photogrammetry data. The method we adopt in the present study is the last one.

Photogrammetry, a photo-based technique for 3D reconstruction, is one of the most cost-effective ways of digitisation. ${ }^{29}$ Photogrammetry has several applications nowadays, including architectural survey, cultural relics digitisation, remote sensing survey and mapping. ${ }^{30}$ However, according to previous studies, ${ }^{27} 3132$ the anatomical use of photogrammetry is still largely limited to the distance measurement of the human organs or anatomical structures. The principle underlying photogrammetry is the computer-assisted reconstruction of digital models from the two-dimensional (2D) photographs of an object captured at various angles. ${ }^{33}$ Unlike the input in the DICOM or 3D scanner, the input in photogrammetry is 2D images, and the typical output is a $3 \mathrm{D}$ model of some real-world object or scene. The whole process is carried out without involving expensive technologies or specific expertise, thus possessing a tremendous potential for producing accurate and affordable 3D models for medical students and teachers.

As for the method of implementing photogrammetry, the first element required is software for data processing. Currently, offline photogrammetry software (eg, Agisoft Metashape; Agisoft, St. Petersburg, Russia) is available for the personal computer, significantly lowering the application threshold of 3D technology for home-made digital models. However, the price is high, the working process could be very time-consuming, and the process largely relies on the computing power of the computer. In the recent past, internet cloud services for photogrammetry have been rapidly expanding, Get3d (www.get3d. cn; Daspatial, Wuhan, China) being an example. The complex computing work can be performed on powerful cloud servers, which can effectively reduce the time taken by photogrammetry.

The second element of photogrammetry is 2D photographs capable of providing enough data for 3D reconstruction. Frequently, high-definition cameras with specific manual settings help to reconstruct precise 3D models and reduce the time required for the alignment of photographs. ${ }^{2732-35}$ There are reports of the multicamera rig being introduced in photogrammetry for higher reconstruction quality. ${ }^{27}{ }^{36}$ However, the shortcomings brought about by the lack of clarity in the photographs can be overcome by increasing the number of photographs. ${ }^{37}$ In our study, we have investigated cloud-based photogrammetry with a phone camera (eight megapixels) and examined the quality of 3D digital models and printings obtained.

Until now, 3D imaging and printing have been generally known to require professional knowledge and special equipment. In the present study, we have attempted to bring together phone-based 3D imaging, cloud-based photogrammetry and affordable 3D printing in an entirely new workflow to aid anatomical study, particularly in low-income and middle-income countries.

\section{Bone specimens}

The skeletal specimens of a 62-year-old Han ethnic male used in this study were provided by the Morphologic Science Experimental Center, School of Basic Medical Science, Central South University. An eight-megapixel phone camera (iPhone SE; Apple, Cupertino, California, 


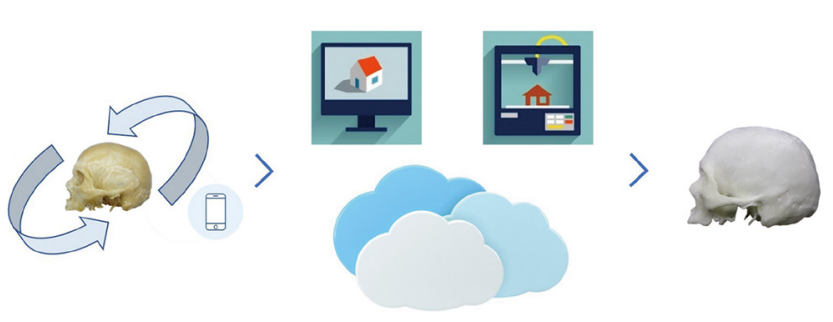

3D Photographing

Cloud Services

3D Printing Mode

Figure 1 Flow chart of technical route. Three main stages are involved. first, specimens acquired are photographed from all around to obtain enough 2D images from all possible directions. Second, 2D images are converted into digital models with a cloud-based specialised server. Third, after editing, digital models and 3D printing setting data are applied to 3D printer for printing. 2D, two dimensional; 3D, three dimensional.

USA,) was used for capturing images. The data upload to and download from a cloud server (www.get3d.cn; Daspatial) were carried out through the Internet. Considering both the morphological classification and the features of the bones, we studied typical bone specimens-the femur, rib, cervical vertebra and skull to investigate the applicability of our workflow. As the femur is the longest bone, we attempted to build a full-scale replica using the segmentation method; this could be a possible solution to the problems that have been reported involving the printing of specimens larger than the build tray of the printer. ${ }^{9}{ }^{38}$ Further, we chose the cervical vertebra and skull to test the capability of our workflow in building small-scale, irregular replicas and large, complex replicas, respectively. Finally, since the reconstruction and production of an object with curved surfaces was considered a challenge in manufacturing, ${ }^{39}$ we studied the performance of our workflow in making a replica of the rib. The printing was done by an online service provider (www.wenext.cn; WeNext Technology, Shenzhen, China) who used the 3D printer RSPro 450 (Union Technology, Shanghai, China) and Somos Imagine 8000 (SI8000; DSM Desotech, Illinois, USA) printing material. The general process of the workflow is summarised in figure 1.

\section{Photographing of bone specimens}

To start with, the specimen was placed on a turntable with a calibration pattern on a plain background. With even lighting from all sides, the specimen was photographed repeatedly using a phone camera. The object should occupy a significant portion of each image, and each part of the specimen should be captured by at least two pictures to be 3D reconstructed. For this, approximately $60 \%-80 \%$ overlap in succeeding pictures is recommended. During our testing, the photographer held the phone and captured the images with one hand and rotated the turntable with the other hand after each shot. Two rounds of photography were carried out on different horizontal planes. In the first round, the dip angle from the phone camera to the specimen was set at $30^{\circ}$, the photographs were captured stepwise by rotating the specimens by $15^{\circ}$ each time or by a lower angle in the cases where complex/fine structures were encountered. For example, when photographing the base of the skull, the specimen was rotated through $5^{\circ}$ between consecutive filming. In the second round, the dip angle was set at $60^{\circ}$ and all operations were performed similarly. In the process of photo acquisition, it is necessary to capture multiple orientations by flipping the specimens to capture any missing surfaces. The whole process yielded $80-100$ or different images for each specimen, requiring approximately 8 min per model. Because of the differences in the size, shape and structural complexity of the specimens, the timing and direction of photography were set more or less different for the individual specimens. Thus, for the rib and cervical vertebra, 80 shots each were captured, whereas, for the femur, 100 shots were captured. As the structure of the skull is highly complex, 120 shots were captured. The resolution of a single photo was $4032 \times 3024$ pixels, with a file size of $\sim 2.5 \mathrm{MB}$.

\section{D assembly of digital images}

The photos captured in the manner described were uploaded to the Get3d website, which is a free cloudbased photogrammetric server providing 3D reconstruction services. Thus, a 3D model file (in .obj format) was generated, following the integration of the original bone photos and the creation of texture information. Next, the unnecessary objects created along with the specimens from the background of the photos were deleted using the mesh editing software Materialise Magics 22 (Materialise NV, Technologielaan, Leuven, Belgium). The free and open-source software MeshLab 2016 is also capable of doing this. Moreover, an 'Automatic Fixing' tool was used here to repair the corrupt digital models. Furthermore, to make the dimensions of the digital model consistent with that of the real specimen, the 'Measurement' and 'Scale' tools were used to adjust the pixel distance between two randomly chosen points on the digital models to the physical distance on the specimen measured using Vernier callipers. Finally, a 1:1 digital model was exported in stereolithography (.Stl) file format. For the long specimen (ie, the femur), separate digital models were made for its different parts and then were digitally merged to produce the digital model of the complete specimen in Geomagic Control 2015 (3D Systems, Morrisville, North Carolina, USA).

\section{D printing}

To reduce the use of printing materials, the wall thickness of each digital model was set to $2 \mathrm{~mm}$ on the interface. The files $(50-100 \mathrm{MB})$ were then submitted for online 3D printing services. For the printing process, the femur, rib, vertebra and skull consumed 136 018.93, 26 530.71, 7550.15 and $237720.08 \mathrm{~mm}^{3}$ of SI8000, respectively. The total cost of the 3D printing of all the models was USD 60.76 , with the material fee accounting for $30 \%$ and the services for $70 \%$ of the total costs. The costs of the 3D 
printed femur, rib, cervical vertebra and skull were USD $20.27,3.96,1.13$, and 35.40 , respectively.

\section{Evaluation of digital and printed models with respect to bone specimens}

The quality of the 3D printed models could be affected by several factors involved in the process of photography, 3D digitalisation and 3D printing. Therefore, to determine the fidelity, we carried out both the identification of morphological features and morphometric analysis among the digital model, 3D printed model and corresponding specimens using Geomagic Control 2015.

The anatomical features on the 3D printed models and digital models relative to the original specimens were analysed with reference to the Atlas of Human Anatomy, sixth edition. ${ }^{40}$ Specifically, the number and appearance of annotated anatomical structures in the book were identified and assessed on the printed models relative to the specimens by experienced anatomy teachers.

For the deviation analysis, bone specimens and 3D printed models were scanned with a 3D scanner to create their digital files. The 3D scanner used was OKIO-5M400yy light scanner (TenYoun 3D Technology, Beijing, China). The scanned files were all in (.stl) file format. Thereafter, the deviations among the three digital models (ie, scanned models of 3D printed models, scanned models of specimens and phone-photometric digital models) were compared using Geomagic Control 2015. When evaluating the performance of photogrammetry for digitisation, the scanned models of the specimens were defined as the references, whereas the phonephotometric digital models were used as the tested counterparts. Similarly, when evaluating the accuracy of the 3D printing, the phone-photometric digital models were defined as the references, whereas the scanned models of the 3D printed models were tested. After the matched models were selected, the software first generated thousands of sample points all over the surfaces of both the models evenly for deviation counting. Thereafter, the software overlapped the two models in space until an optimal overlap was achieved. Next, the local Euclidean distances (EDs) between pairs of corresponding sample points on the two surfaces were calculated, and the deviations were represented as diagrams and illustrated as heat maps.

\section{Patient and public involvement}

The bones of a deceased body donor were used for 3D imaging and printing in the present study. The body donor has agreed to be involved in medical research and education in the future. The next kin of the body donor has agreed on the planning, design and implementation of this study, and has signed written consent. Regarding the results of 3D imaging and printing, the next kin of the donor was satisfied with the outcomes and thought this workflow could better promote the benefits of body donors for anatomy education.

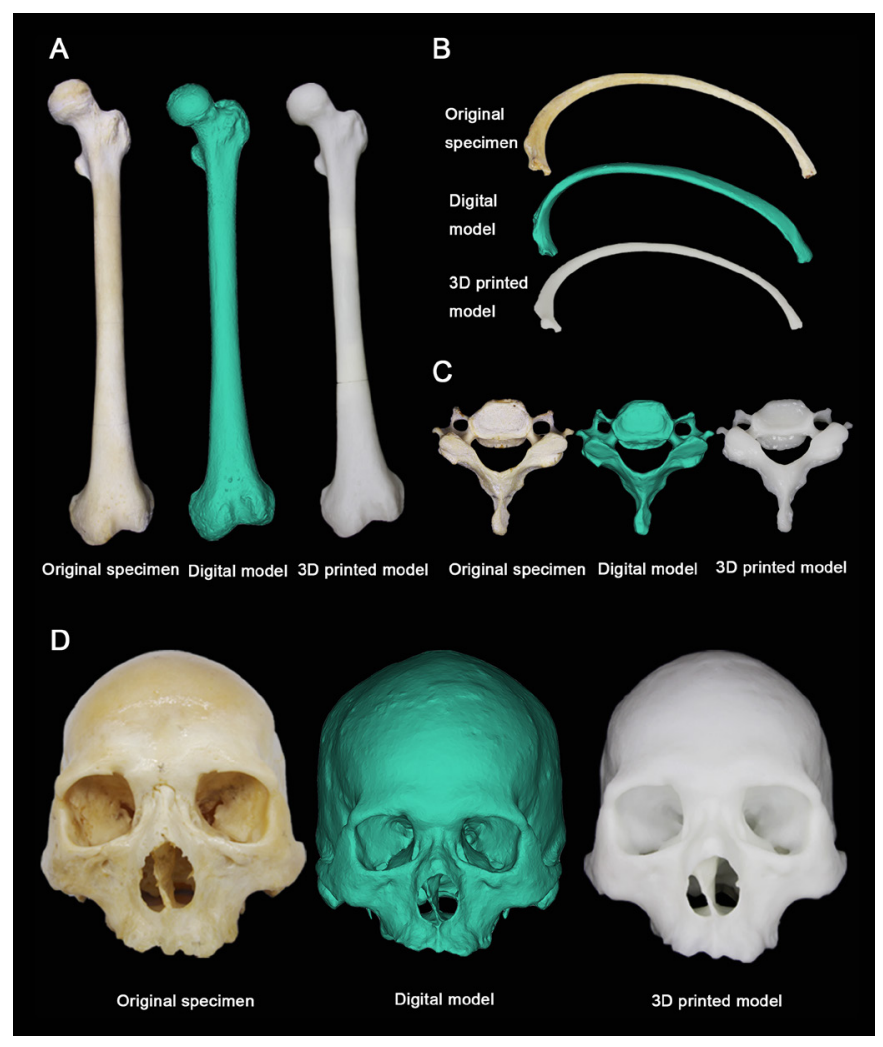

Figure 2 Original specimens, digital models and 3D printed models made with SLA technology. (A) femur, (B) rib, (C) cervical vertebra, (D) skull (the digital models may seem smaller because of the special display mode in materialise magics, which is different from single perspective). 3D, threedimensional; SLA, stereolithography apparatus.

\section{RESULTS}

\section{Gross anatomical analysis of digital and printed models relative to specimens}

The overall morphology of the digital and 3D printed models displayed a high extent of similarity to the corresponding specimens from the gross anatomical perspective, as depicted for the femur, rib, cervical vertebra and skull (figure 2). By further examination, the existence and details (textures, smoothness and shadows) of the various bone structures, such as protrusions, ridges, lines, sutures and foramina/aperture, appeared to be largely presented on the digital and printed models (figure 3). Using Netter's Atlas of Human Anatomy as a reference, we attempted to identify as many anatomical structures as possible on each set of digital and 3D printed models relative to the specimens (online supplementary figure S1-S4); data collected from the four different bones are listed in table 1 and online supplementary table S1 and also discussed here.

Both the digital and 3D printed models of the femur retain 27 anatomical features of those defined in Netter's Atlas of Human Anatomy. Large-sized anatomical structures, such as the great trochanter, lateral condyle, linea aspera, were always presented, and a vast majority of fine features (eg, intertrochanteric crest) were also identifiable. However, structures characterised by low resolution 


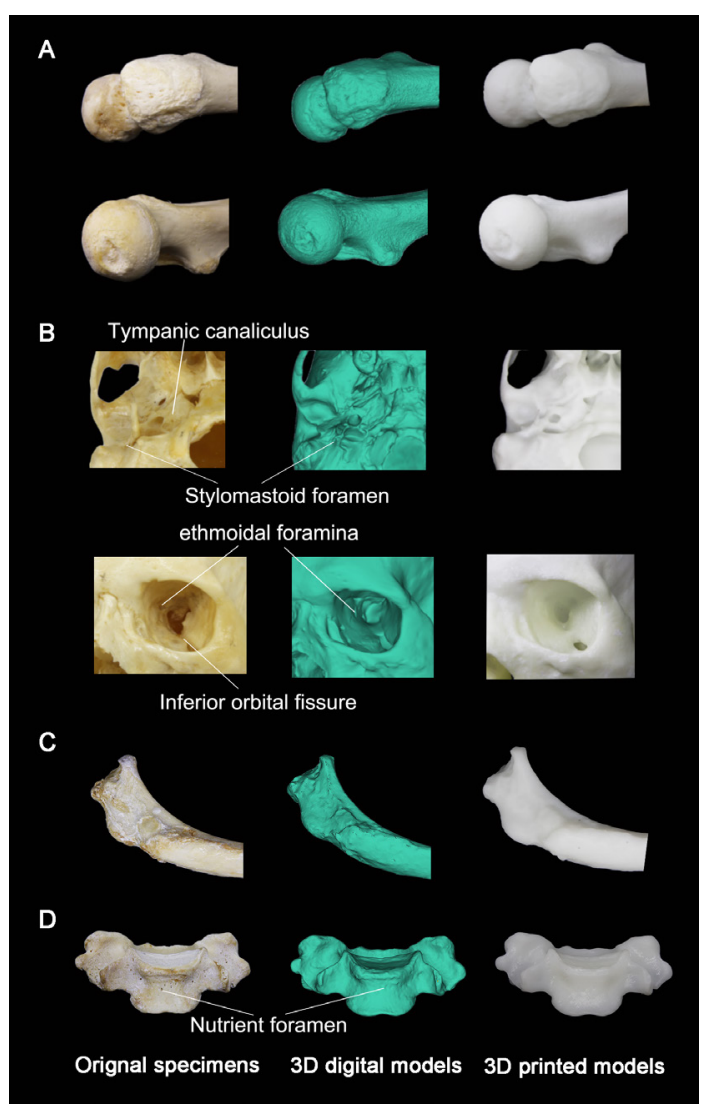

Figure 3 Comparison of fine structures among specimens, digital models and prints. (A) Nutrient foramina in the great trochanter (above) and the fovea for ligament of head (below). (B) Nerve foramina in cranial base (above) and intraorbital structures (below). (C) Tubercle of the rib. (D) Nutrient foramina in vertebral body.

(eg, the pectineal line) visible on the physical specimens and digital models were not fully reflected on the 3D printed models. The nutrient foramina and retinacular foramina were partly lost. For the rib specimen, eight features were attempted to be identified on the digital and 3D printed models. Among them, only the inferior articular facets of the vertebral body were not apparent enough on both models relative to the specimen. For the cervical vertebra, all 10 anatomical features defined in Netter's Atlas were presented in both the digital and print models (table 1 ).

A total of 103 anatomical features were macroscopically examined and identified on the skull specimen with reference to Netter's Atlas. Of these, 96 were found on the digital model and 92 were found on the print model (figure 4; online supplementary table S1). Overall, the large-sized features (eg, the frontal bone, maxilla and temporal bone) were more distinctly reproduced and correctly proportioned relative to those of smaller size. However, features that were flat and small (eg, the asterion, bregma and lambda) could not be reliably found on either the digital model or the 3D print. This was also the case for some difficult-to-photograph fine-scale structures (eg, the zygomaticofacial foramen, ethmoidal foramina, stylomastoid foramen and tympanic canaliculus). Several other structures, including the tympanic canaliculus, lesser palatine foramina, pterygoid hamulus and ethmoidal foramina, were also not well resolved on the digital model owing to a lack of photographic minuteness and shallowness (ie, poor contrast). Further, it should be noted that a number of shallow sutures, small holes, or thin bony plates were not well represented on the 3D printed model, most likely because the resolution of the printer was not high or precise enough (figure 3; online supplementary figure S5-S7).

Of particular interest was the extent of reproduction of the nutrient foramina on the models. In the femur bone, 205 recognisable nutrient foramina were identified. However, the number dropped to 131 on the digital model and 117 on the printed model. The dimension of the foramina was the single most determinant factor related to the reproduction of the foramina on the models. Those with a diameter of $<0.5 \mathrm{~mm}$ were unrecognisable on the digital model and those with a diameter of $<1.20 \mathrm{~mm}$ were unrecognisable on the 3D printed model. Moreover, the nutrient foramina at a depth $>1.5 \mathrm{~mm}$ in the specimen were found at depths $0.2 \pm 0.1$ and $0.4 \pm 0.1 \mathrm{~mm}$ shallower, respectively, in the digital and $3 \mathrm{D}$ printed models.

To summarise, loss of quality occurs generally in both the photogrammetry and $3 \mathrm{D}$ printing processes, which is more clearly manifested in the indistinguishability of the small or flat anatomical features. Our assessment indicates that this effect is more evident in the 3D printed models of large-sized specimens. We speculate that this is because small-sized anatomical features take up a relatively small proportion of a large specimen relative to those on a smallsized specimen and, therefore, lack sufficient resolution in 2D photographs. Thus, photos with inadequate data can lead to loss of anatomical details in the final replica.

\section{Morphometric deviation analysis among specimens, digital models, 3D printed models}

Geomagic Control 2015 was used to evaluate the morphometric deviations among specimens, digital models and 3D printed models. According to an estimation of the comparison of fine structures, nutrient foramina, the smallest named anatomical features of all, had a resolution of approximately $0.5 \mathrm{~mm}$ in the digital models and $1.2 \mathrm{~mm}$ in the 3D printed models. Thus, deviations less than $0.5 \mathrm{~mm}$ should not affect the anatomical characteristics, in theory. Therefore, we set $0.5 \mathrm{~mm}$ as the precision range and $2.0 \mathrm{~mm}$ as the tolerance range. The deviations were depicted in diagrams and illustrated as heat maps, in which hot colour represented positive deviations (ie, the EDs were greater than $0.5 \mathrm{~mm}$ ), cool colour represented negative deviations (ie, the EDs were less than $-0.5 \mathrm{~mm}$ ) and green colour indicated deviations within the precision range.

The comparison between specimens and 3D printed models was aimed to estimate the overall deviations that occurred during the whole workflow (figures 5 and 6; online supplementary table S2). For the femur, the deviations of $88.39 \%$ of the sample points were less than 
Table 1 Extent of reproduction of anatomical features in digital and 3D printed models of bone specimens.

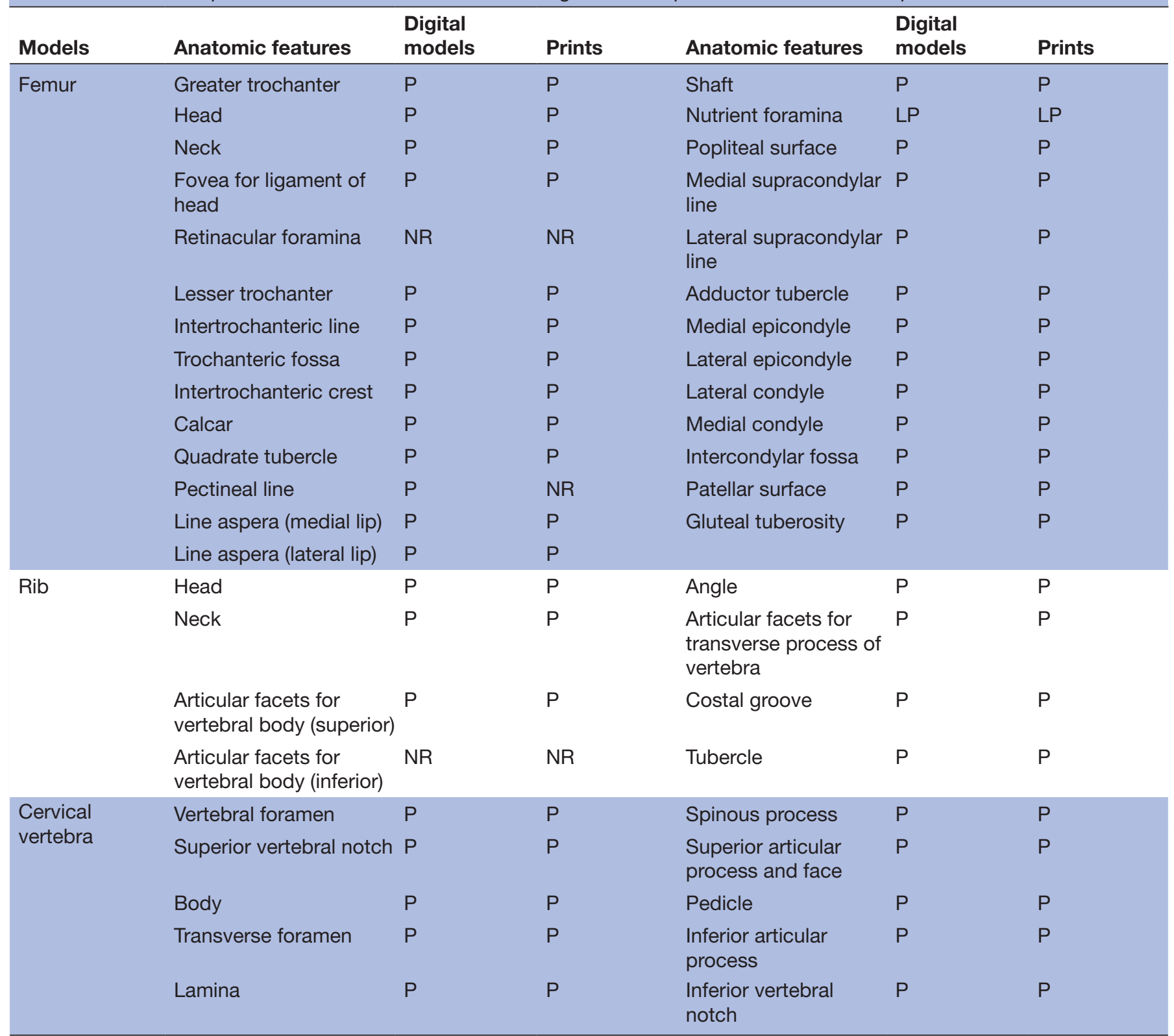

Atlas of Human Anatomy, sixth edition (edited by Frank $\mathrm{H}$ Netter) was used as a reference book for anatomical annotations, with all listed anatomical features observed on in original bone specimens.

3D, three-dimensional; LP, lost partly; NR, not well resolved; P, present distinctly.

$0.5 \mathrm{~mm}$, and those of $99.316 \%$ were less than $2 \mathrm{~mm}$. Both mean positive and negative deviations were less than $0.5 \mathrm{~mm}$, and only some positive deviations could be seen for both ends of the femur (figure 5A). For the rib, the deviations of $83.26 \%$ of the sample points were less than $0.5 \mathrm{~mm}$, and those of $99.16 \%$ were less than $2 \mathrm{~mm}$. Positive deviations were seen mainly for the lateral cartilaginous end and the head of the rib, whereas negative deviations were seen mainly for the upper edge and medial cartilaginous end (figure 5B). For the cervical vertebra, the deviations of $99.72 \%$ of the sample points were less than $0.5 \mathrm{~mm}$, and those of $99.99 \%$ were less than $2 \mathrm{~mm}$ (figure 5C). Neither the positive nor the negative deviations were apparent in the heat map deviation spectrum.
Distributions of their deviations were shown in figure $5 \mathrm{D}$. For the skull, the deviations of $85.69 \%$ of the sample points were less than $0.5 \mathrm{~mm}$, and those of $99.34 \%$ were less than $2 \mathrm{~mm}$ (figure 6). Generally, the deviations were concentrated more in uneven areas, particularly in parts with larger curvatures, such as deep and narrow cavities or processes. Low positive deviations were scattered in the parts of the cranial base. High positive and negative deviations were distributed mainly for the styloid process, optic canal, choanae and foramina in the cranial base.

The comparison between the digital and 3D printed models was aimed at determining the degree of deviation caused in the process of 3D printing after the generation of the digital models, which could be attributed to the 
3D Printed Model

Digital Model
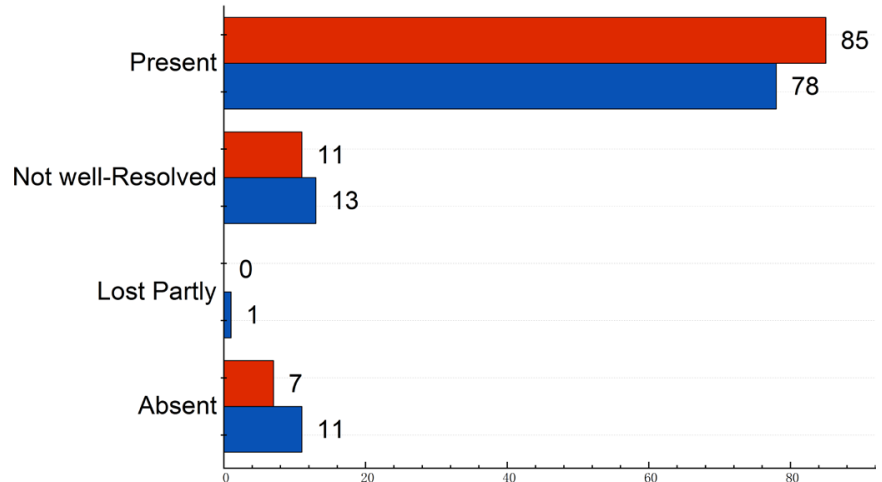

Figure 4 Comparison of anatomical features' retention in digital model and 3D printed models of skull. Atlas of Human Anatomy, sixth edition (Frank H. Netter, 2015) was used as a reference book for anatomical annotations. all the features chosen are well-observed in the original specimen. 3D, threedimensional.

quality/resolution of the printer and/or the printing materials (online supplementary table S3). The relevant data were collected and presented and are illustrated in figure 7 . For the femur, the deviations of $99.45 \%$ of the sample points were less than $0.5 \mathrm{~mm}$, and those of $99.71 \%$ were less than $2 \mathrm{~mm}$. According to the heat-map deviation spectrum, green colour covered the entire surface, whereas the positive and negative deviations were not evident (figure 7A). For the rib, the deviations of $90.24 \%$ of the sample points were less than $0.5 \mathrm{~mm}$, and those of

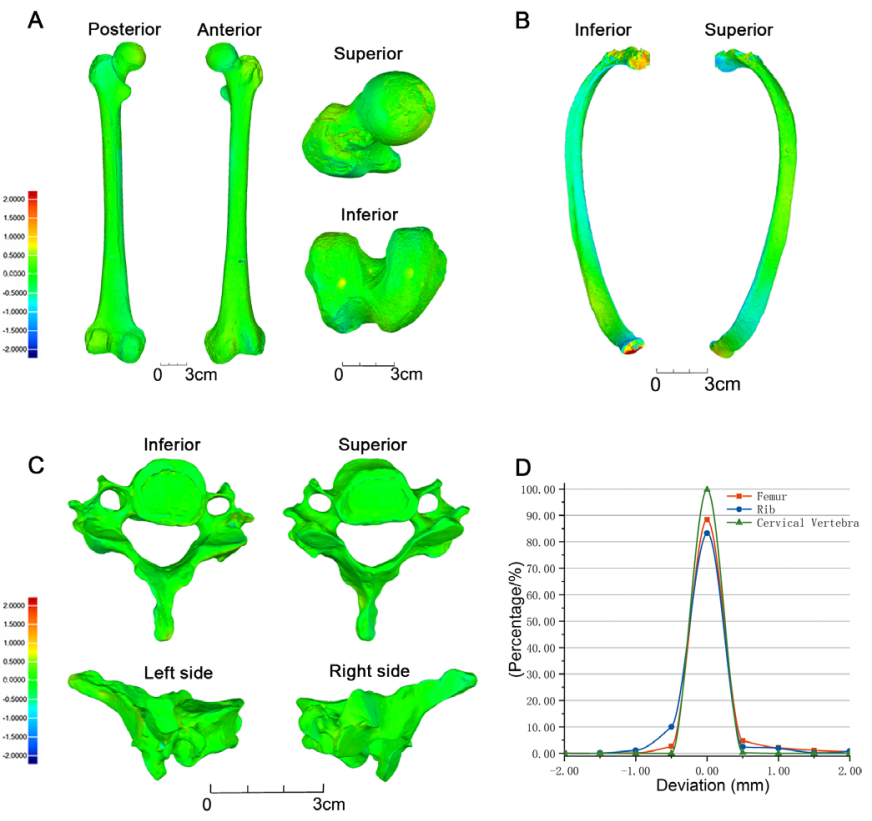

Figure 5 Analysis of deviation between original specimens and 3D printed models. Gradation on the deviation spectrum is $0.5 \mathrm{~mm}$ each; green colour indicates deviations ranging from -0.5 to $0.5 \mathrm{~mm}$; hot colour indicates positive deviations ranging from 0.5 to $2 \mathrm{~mm}$; cool colour indicates negative deviations ranging from -0.5 to $2 \mathrm{~mm}$. deviation analysis of (A) femur, (B) rib, (C) cervical vertebra; (D) distribution of deviations (in \%). 3D, three-dimensional.
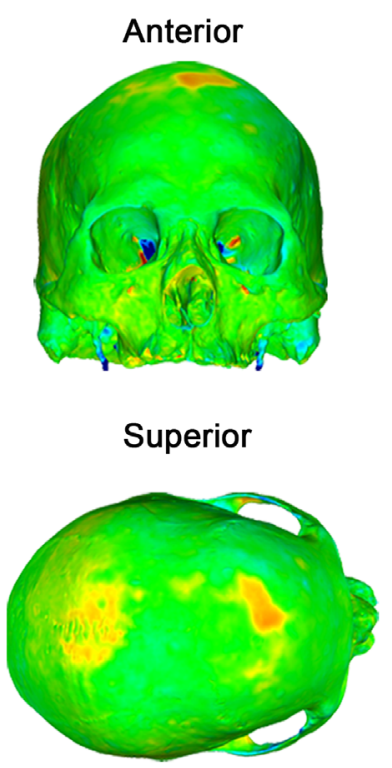

Right side
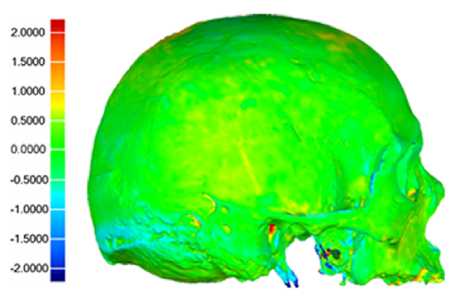

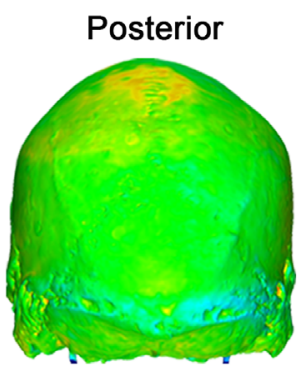

Inferior
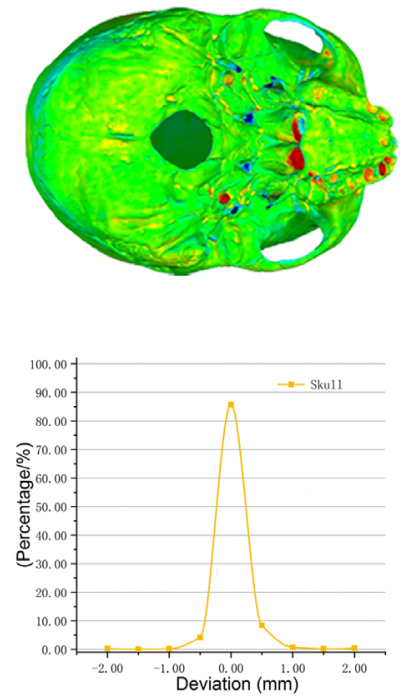

$0+3 \mathrm{~cm}$

Figure 6 Analysis of the deviation between original cranial specimens and 3D printed skull. Gradation on the deviation spectrum is $0.5 \mathrm{~mm}$ each; green colour indicates deviations ranging from -0.5 to $0.5 \mathrm{~mm}$; hot colour indicates positive deviations ranging from 0.5 to $2 \mathrm{~mm}$; cool colour indicates negative deviations ranging from -0.5 to $2 \mathrm{~mm}$. Distribution of deviations is displayed in the histogram (in \%). 3D, threedimensional.

all the sample points were less than $2 \mathrm{~mm}$. The mean positive and negative deviations were distributed evenly on the body of the rib. High positive and negative deviations were seen mainly for both ends of the rib (figure 7B). For the cervical vertebra, the deviations of $99.84 \%$ of the sample points were less than $0.5 \mathrm{~mm}$, whereas those of $99.99 \%$ were less than $2 \mathrm{~mm}$. On the heat-map deviation spectrum, both positive and negative deviations were not apparent (figure 7C). For the skull, the deviations of $97.88 \%$ of the sample points were less than $0.5 \mathrm{~mm}$, and those of $99.59 \%$ were less than $2 \mathrm{~mm}$. High positive deviations were scattered in a limited region near the optic canal, whereas low negative deviations were scattered on the occipital bone region. The lateral pterygoid plates also indicated deviations owing to defects in the 3D printed models (figure 7D). Distribution of the deviation was shown in figure 7E.

In the final analysis, all the deviations generated in the entire workflow were practically within the tolerance 

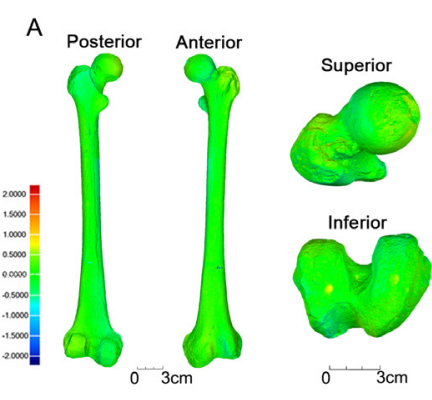

B
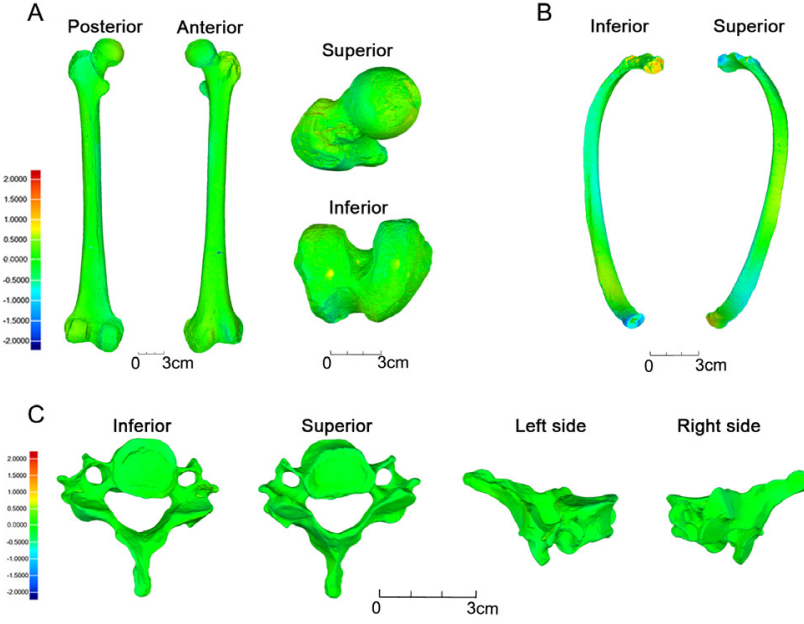

D

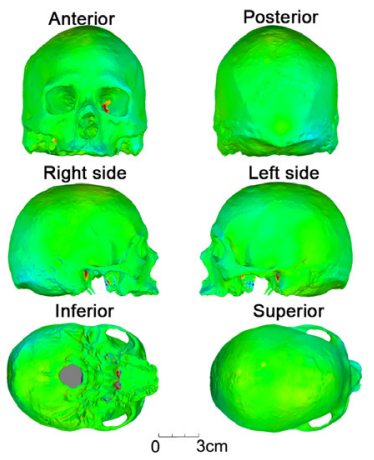

$E$

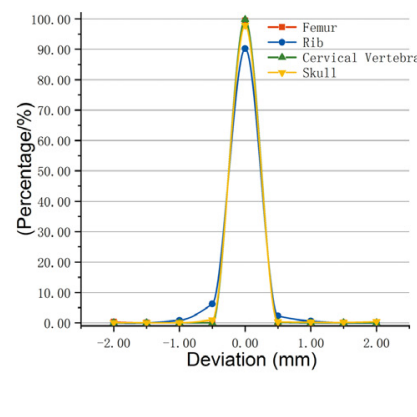

Figure 7 Analysis of the deviation between digital models and 3D printed models. Gradation on the deviation spectrum is $0.5 \mathrm{~mm}$ each; green colour indicates deviations ranging from -0.5 to $0.5 \mathrm{~mm}$; hot colour indicates positive deviations ranging from 0.5 to $2 \mathrm{~mm}$; cool colour indicates negative deviations ranging from -0.5 to $2 \mathrm{~mm}$. deviation analysis of (A) femur, (B) rib, (C) cervical vertebra; (D) skull; (E) distribution of deviations (in \%). 3D, three-dimensional.

range, mainly distributed in the precision range, which could be considered to have little impact on the resolution. In most cases, the deviations are mainly generated in the photogrammetry process instead of in the printing process. However, both the photogrammetry and printing of the rib generate deviations that cannot be ignored. The result is in accordance with the point of view that there still exist challenges in producing objects with curved surfaces. ${ }^{39}$ Furthermore, we may conclude that curvature also has an impact on the photogrammetry process.

\section{DISCUSSION}

The photogrammetric digitisation method adopted in the present study demonstrates a fairly high precision of the digital and 3D printed models. The most noteworthy feature of the proposed workflow is that it works without scanners or the CT/MRI dataset, thus enabling a broader range of 3D printing technology for educational applications.

Further, the photogrammetry method used in this study for digitisation is advantageous for capturing the surface structures of an object so that they can be reproduced accurately. Because the digital models generated using the photogrammetry method have textures and maps, not only the shape of but also the light and the shadow on the surface could be generated using the 3D models, making the models more realistic and easier to edit. ${ }^{32} 33$ Based on these characteristics, phone camera-based photogrammetry is also expected to be applied in field research projects, in which 3D digital data on human or animal bone specimens or other remains have to be quickly obtained. The 3D printed models created using the photogrammetry method demonstrate only the external features of the bone specimens; the inner structures are invisible. Human specimens also have this limitation. To display the different anatomical landmarks on the interior of the skull, three or four differently dissected specimens must be used. The same strategy can be applied while creating 3D printed models that display different anatomical structures-differently dissected or sliced specimens are chosen as the resources to be put through photogrammetry. On the other hand, although images from CT/ MRI data for 3D reconstruction is a more preferred means for the 3D reconstruction of internal structures, it still involves challenges such as high cost, low fidelity and colourless images. ${ }^{22}$ Further, although the raw digital models built using the photogrammetry method retain the original colour, the use of multicolour printing material or facilities leads to increased costs. Therefore, we used single-colour material to print.

The cloud server we adopted generated high-resolution digital models quickly, which can be used for mass production, and was fairly easy to use. Comparing the DICOM images from CT/MRI data, we find that limitations still exist because specialised skills are required to operate the system to separate target organs for printing; also, DICOM data for 3D reconstruction are occasionally imprecise owing to the constant organ movements of the living subject. ${ }^{38}$ Moreover, the cloud service for 3D modelling is free to use, and it can save at least US $\$ 179$ compared with the offline software (eg, Agisoft Metashape costs US $\$ 179$ for the standard edition and US\$3499 for the professional edition). To control the use of resins and the cost, the 'hollowing' function can be used in the model editing process to adjust the wall thickness of the model to a smaller degree. To further lower the cost, the free and open-source software MeshLab can be used for editing the digital model. These costeffective strategies can help promote the application of $3 \mathrm{D}$ printing programme in medical education in India and Brazil. However, this workflow is not applicable to medical schools in the countries that have no internet connection, although the dataset needed to upload to cloud services is not large.

For the specimens that are larger than the build tray of the printer, the scaling down method is often adopted. ${ }^{9}$ However, the drawbacks of this method cannot be neglected. The resolution of a rescaled 3D printed model decreases and some anatomical details might be lost in 
the process. In this study, the digital models of the femur were divided using the software, and cylindrical structures were incorporated on the cross section of the model, so that the 3D printed parts could be manually joined together. Using this method, the problem of printer adaption could possibly be addressed and the integrity of the specimens can be maintained.

Finally, the internet 3D printing platform and SLA printing technology were selected. According to a report, the printing quality of the SLA is higher than that of FDM, SLS, LOM or binder jetting (BJ), attaining the same level as that of polyjet (PJ) ${ }^{24}$ In addition, the overall cost for printing an object with SLA is average, which is higher than those with FDM, LOM and BJ but lower than those of SLS and PJ. ${ }^{24}{ }^{25}$ The results of our study indicated that the 3D printed models could well display the anatomical features, and the nutrient foramina on the femur could be observed easily. However, in another study involving femoral 3D printing using Acrylonitrile butadiene styrene (ABS) in FDM technology, the nutrient foramina on the printed femur were reported to be invisible. ${ }^{23}$ The printing material used in this study was Somos Imagine 8000, which conforms to USP Class IV and International Organization for Standardization (ISO) 10993 international standards. It is safe and has excellent physical properties and antifouling performance, making the printed models dense, wear-resistant, impact-resistant and easy to clean. The Somos Imagine 8000 is rigid and is not suitable for 3D printing skins and muscles in comparison with soft material (eg, thermoplastic polyurethanes).

Laboratory dissection and dissected specimens are essential for learning the 3D concept of the structure of the human body. ${ }^{41}$ In the past, this anatomical learning was limited by a lack of human specimen resources owing to the insufficiency of body donation in several countries. The primary advantage of 3D printing applied in anatomical education is its ability to create models, which can demonstrate the high complexity of human body in great detail. Additionally, the 3D printed models have no unpleasant smell and are easier to clean and preserve than the embalmed cadaveric specimens. Moreover, it was reported that students who have been educated with 3D printed models performed better in tests than those educated with human specimens. ${ }^{23}$ Although relatively cheap injection models made of plastic powders (eg, $\sim$ US $\$ 30$ for injection model of the skull; US $\$ 35$ for the printed model of this study) were used as substitutes for original specimens, several problems were overlooked. First, before the production of the injection models, the corresponding moulds are to be made using standard data. The anatomical structures are universal but found to be lacking in the natural specificity and diversity. Thus, they cannot display the structural variations and pathological changes of human specimens. On the other hand, the manufacturing process of the plastic models and moulds is complicated. These can only be produced by professional companies and not by the teaching staff in medical schools. This issue may be solved using the photogrammetry method. Students and teachers can take pictures of the specimens in class and convert them into digital models, particularly specimens of various ages, pathological conditions and disease processes. ${ }^{25}$ In this manner, they can view and manipulate digital models using their mobile phones and even set up private digital libraries. Further, teachers and students can also establish a characteristic database of 3D digital models based on their own specimens using the cloud-based photogrammetry method. It is a means to permanently store some specimens with historical significance, such as foot-bone specimen of the 'Three-inch Golden Lotus', a vile feudal practice that crippled young girls both physically and spiritually in feudal China. As a cutting-edge technology, it is believed that 3D printing can be comprehensively improved in terms of advanced materials and affordable prices in future applications in anatomical education.

\section{CONCLUSIONS}

In this study, a workflow composed of phone camera photography, cloud-based photogrammetry and online 3D printing was introduced to make customised replicas of bone specimens with different sizes and complexities. According to statistics, both the digital and 3D printed models have relatively high precision and retain most of the anatomical features. Using phone camera-based photogrammetry, cloud-processing services for 3D modelling and the hollowing method to control the use of resins, this technical route remarkably facilitates the production of personalised 3D digital models and prints, in a cost-effective manner for both teachers and students.

Acknowledgements The authors thank the body donor and the next kin for supporting anatomical education. The authors also thank Prof. Xiao-Xin Yan for critical reading.

Contributors $Q Z$ and $Q-Y L$ designed the project. $Q Z$ and $Y H$ provided specimens. A-HP and FL got grants. Q-YL and CY imaged and printed $3 D$ models. Q-YL, CY, MP, QZ wrote and revised the paper. All authors read and proved the manuscript.

Funding This work was supported by grants from the National Natural Science Foundation of China (81471372, 91632116), the Natural Science Foundation of Hunan Province (2018JJ3635) the Research Project on Teaching Reform in Hunan Province (2018-57), the Technology Innovation Guide Program of Hunan Province (2018ZK4031), the Research Project on Teaching Reform for Academic Degrees and Graduate Education in Hunan Province (JG2018B021), the Research Project on Teaching Reform for Undergraduate Education in Central South University (2018jy135, 2019jy136), and the Research Project of Laboratory Construction and Management in Central South University (201807).

Competing interests None declared.

Patient consent for publication Next of kin consent obtained.

Ethics approval The study was approved by the Ethics Committee of the Third Xiangya Hospital, Central South University (Ethical Application Ref: 2019-S243).

Provenance and peer review Not commissioned; externally peer reviewed.

Data availability statement Data are available on reasonable request.

Open access This is an open access article distributed in accordance with the Creative Commons Attribution Non Commercial (CC BY-NC 4.0) license, which permits others to distribute, remix, adapt, build upon this work non-commercially, and license their derivative works on different terms, provided the original work is properly cited, appropriate credit is given, any changes made indicated, and the use is non-commercial. See: http://creativecommons.org/licenses/by-nc/4.0/. 
ORCID iD

Qi Zhang http://orcid.org/0000-0001-6300-6491

\section{REFERENCES}

1 Memon I. Cadaver dissection is obsolete in medical training! a misinterpreted notion. Med Princ Pract 2018;27:201-10.

2 Sugand K, Abrahams P, Khurana A. The anatomy of anatomy: a review for its modernization. Anat Sci Educ 2010;3:83-93.

3 AbouHashem Y, Dayal M, Savanah S, et al. The application of 3D printing in anatomy education. Med Educ Online 2015;20:29847.

4 Chen D, Zhang Q, Deng J, et al. A shortage of cadavers: the predicament of regional anatomy education in mainland China. Anat Sci Educ 2018;11:397-402.

5 Shui W, Zhou M, Chen S, et al. The production of digital and printed resources from multiple modalities using visualization and threedimensional printing techniques. Int $J$ Comput Assist Radiol Surg 2017;12:13-23.

6 Zhang Q, Deng J, Li Y-N, et al. Perceptions and attitudes toward brain donation among the Chinese people. Anat Sci Educ 2020;13:80-90.

7 Ghosh SK. Cadaveric dissection as an educational tool for anatomical sciences in the 21st century. Anat Sci Educ 2017;10:286-99.

$8 \mathrm{Li}$ Z, Li Z, Xu R, et al. Three-Dimensional printing models improve understanding of spinal fracture $-A$ randomized controlled study in China. Sci Rep 2015;5:11570.

9 McMenamin PG, Quayle MR, McHenry CR, et al. The production of anatomical teaching resources using three-dimensional (3D) printing technology. Anat Sci Educ 2014;7:479-86.

10 Vaccarezza M, Papa V. 3D printing: a valuable resource in human anatomy education. Anat Sci Int 2015;90:64-5.

11 Lee H, Fang NX. Micro 3D printing using a digital projector and its application in the study of soft materials mechanics. J Vis Exp 2012:e4457.

12 Mogali SR, Yeong WY, Tan HKJ, et al. Evaluation by medical students of the educational value of multi-material and multi-colored three-dimensional printed models of the upper limb for anatomical education. Anat Sci Educ 2018;11:54-64.

13 Rengier F, Mehndiratta A, von Tengg-Kobligk H, et al. 3D printing based on imaging data: review of medical applications. Int J Comput Assist Radiol Surg 2010;5:335-41.

14 Andolfi C, Plana A, Kania P, et al. Usefulness of three-dimensional modeling in surgical planning, resident training, and patient education. J Laparoendosc Adv Surg Tech A 2017;27:512-5.

15 Bannon R, Parihar S, Skarparis Y, et al. 3D printing the pterygopalatine fossa: a negative space model of a complex structure. Surg Radiol Anat 2018;40:185-91.

16 Chou P-Y, Hallac RR, Shih E, et al. 3D-Printed models of cleft lip and palate for surgical training and patient education. Cleft Palate Craniofac J 2018;55:323-7.

17 Schubert C, van Langeveld MC, Donoso LA. Innovations in 3D printing: a 3D overview from optics to organs. Br J Ophthalmol 2014;98:159-61.

18 de Alcântara Leite Dos Reis D, Gouveia BLR, Júnior JCR, et al. Comparative assessment of anatomical details of thoracic limb bones of a horse to that of models produced via scanning and $3 D$ printing. 3D Print Med 2019;5:13.

19 Jawale YK, Rapol U, Athale CA. Open source 3D-printed focussing mechanism for cellphone-based cellular microscopy. J Microsc 2019;273:105-14.

20 Lim KHA, Loo ZY, Goldie SJ, et al. Use of 3D printed models in medical education: a randomized control trial comparing 3D prints versus cadaveric materials for learning external cardiac anatomy. Anat Sci Educ 2016;9:213-21.
21 Preece D, Williams SB, Lam R, et al. "Let's get physical": advantages of a physical model over 3D computer models and textbooks in learning imaging anatomy. Anat Sci Educ 2013;6:216-24.

22 Cramer J, Quigley E, Hutchins T, et al. Educational material for 3D visualization of spine procedures: methods for creation and dissemination. J Digit Imaging 2017;30:296-300.

23 Li F, Liu C, Song X, et al. Production of accurate skeletal models of domestic animals using three-dimensional scanning and printing technology. Anat Sci Educ 2018;11:73-80.

$24 \mathrm{Kim}$ GD, Oh YT. A benchmark study on rapid prototyping processes and machines: quantitative comparisons of mechanical properties, accuracy, roughness, speed, and material cost. Proc Inst Mech Eng B J Eng Manuf 2008;222:201-15.

25 Garcia J, Yang Z, Mongrain R, et al. 3D printing materials and their use in medical education: a review of current technology and trends for the future. Bmj Stel 2018;4:27-40.

26 Atalay HA, Ülker V, Alkan İlter, et al. Impact of threedimensional printed Pelvicaliceal system models on residents' understanding of Pelvicaliceal system anatomy before percutaneous Nephrolithotripsy surgery: a pilot study. J Endourol 2016;30:1132-7.

27 Kottner S, Ebert LC, Ampanozi G, et al. VirtoScan - a mobile, low-cost photogrammetry setup for fast post-mortem 3D full-body documentations in x-ray computed tomography and autopsy suites. Forensic Sci Med Pathol 2017;13:34-43.

28 Thomas DB, Hiscox JD, Dixon BJ, et al. 3D scanning and printing skeletal tissues for anatomy education. J Anat 2016;229:473-81.

29 Fau M, Cornette R, Houssaye A. Photogrammetry for 3D digitizing bones of mounted skeletons: potential and limits. Comptes Rendus Palevol 2016;15:968-77.

30 Santagati C, Inzerillo L, Di Paola F, et al. Image-Based modeling techniques for architectural heritage 3D digitalization: limits and potentialities. ISPRS - International Archives of the Photogrammetry, Remote Sensing and Spatial Information Sciences 2013;XL-5/ W2:555-60.

31 Nocerino E, Menna F, Remondino F, et al. Application of PHOTOGRAMMETRY to brain anatomy. ISPRS - International Archives of the Photogrammetry, Remote Sensing and Spatial Information Sciences 2017;XLII-2/W4:213-9.

32 Petriceks AH, Peterson AS, Angeles M, et al. Photogrammetry of human specimens: an innovation in anatomy education. J Med Educ Curric Dev 2018;5:238212051879935.

33 Dixit I, Kennedy S, Piemontesi J. Which tool is best: 3D scanning or photogrammetry - it depends on the task. In: Rea PM, ed. Biomedical visualisation :volume 1. Cham: Springer International Publishing, 2019: 107-19.

34 Fu X, Peng C, Li Z, et al. The application of multi-baseline digital close-range photogrammetry in three-dimensional imaging and measurement of dental casts. PLoS One 2017;12:e0178858.

35 Urbanová P, Hejna P, Jurda M. Testing photogrammetry-based techniques for three-dimensional surface documentation in forensic pathology. Forensic Sci Int 2015;250:77-86.

36 Leipner A, Baumeister R, Thali MJ, et al. Multi-camera system for 3D forensic documentation. Forensic Sci Int 2016;261:123-8.

37 Linder W. Digital Photogrammetry: a practical course. 3rd ed. Springer Publishing Company, Incorporated, 2009: 79-80.

38 Smith CF, Tollemache N, Covill D, et al. Take away body parts! an investigation into the use of 3D-printed anatomical models in undergraduate anatomy education. Anat Sci Educ 2018;11:44-53.

39 Hong YZ, Ming YW. The research of contour surface recover technology based on 3D printing parts. Mater Sci Forum 2016;861:182-6.

40 Frank H, Netter M. Atlas of human anatomy. 6th edn. Winsland House I, Singapore: Elsevier Pte., Ltd, 2015.

41 Granger NA. Dissection laboratory is vital to medical gross anatomy education. Anat Rec 2004;281B:6-8. 